\title{
Domain Wall Orientations in Ferroelectric Superlattices Probed with Synchrotron X-Ray Diffraction
}

\author{
Marios Hadjimichael, ${ }^{1, *}$ Edoardo Zatterin, ${ }^{1,2}$ Stéphanie Fernandez-Peña, ${ }^{3}$ Steven J. Leake, ${ }^{2}$ and Pavlo Zubko ${ }^{1}$ \\ ${ }^{1}$ London Centre for Nanotechnology and Department of Physics and Astronomy, University College London, \\ 17-19 Gordon Street, WC1H OAH London, United Kingdom \\ ${ }^{2}$ ESRF-The European Synchrotron, 71 Avenue des Martyrs, 38000 Grenoble, France \\ ${ }^{3}$ Department of Quantum Matter Physics, University of Geneva, CH-1211 Geneva, Switzerland
}

(Received 6 July 2017; revised manuscript received 5 November 2017; published 19 January 2018)

Ferroelectric domains in $\mathrm{PbTiO}_{3} / \mathrm{SrTiO}_{3}$ superlattices are studied using synchrotron x-ray diffraction. Macroscopic measurements reveal a change in the preferential domain wall orientation from $\{100\}$ to $\{110\}$ crystallographic planes with increasing temperature. The temperature range of this reorientation depends on the ferroelectric layer thickness and domain period. Using a nanofocused beam, local changes in the domain wall orientation within the buried ferroelectric layers are imaged, both in structurally uniform regions of the sample and near defect sites and argon ion-etched patterns. Domain walls are found to exhibit a preferential alignment with the straight edges of the etched patterns as well as with structural features associated with defect sites. The distribution of out-of-plane lattice parameters is mapped around one such feature, showing that it is accompanied by inhomogeneous strain and large strain gradients.

DOI: 10.1103/PhysRevLett.120.037602

Recent years have witnessed a renewed interest in ferroelectric domains and domain walls (DWs), particularly at the nanoscale, where DW densities are large and their properties contribute greatly to the overall functional behavior of the materials [1,2]. For example, DW motion is known to greatly influence the macroscopic properties of ferroelectrics, such as their response to an external electric field or external stress [3], and recently has been shown to give rise to the phenomenon of negative capacitance [4,5]. DWs also exhibit symmetry and hence properties that are different from the bulk material. Striking examples include the conducting DWs in insulating multiferroic $\mathrm{BiFeO}_{3}[6,7]$ and ferromagnetic DWs in $\mathrm{TbMnO}_{3}$ [8]. Such properties make multidomain ferroelectrics an attractive pathway towards novel device implementations being explored by the growing field of DW nanoelectronics [1]. The development of macroscopic methods to control the formation and orientation of DWs is thus central to making device fabrication easier using conventional growth and patterning methods.

Ferroelectric superlattices (periodic repetitions of ferroelectric-dielectric bilayers) are ideal systems for the study of ferroelectric domains. The domains in these systems have well-defined periodicities, making them particularly well suited for X-ray diffraction studies [9]. In the past, such superlattices have been used to investigate electrostatic

Published by the American Physical Society under the terms of the Creative Commons Attribution 4.0 International license. Further distribution of this work must maintain attribution to the author(s) and the published article's title, journal citation, and DOI. coupling through dielectric layers [10-12], DW motion under an applied electric field [13,14], improper ferroelectricity [15], and ferroelectric vortices [16].

In this Letter, we present a synchrotron $\mathrm{x}$-ray diffraction study of DW orientations in $\mathrm{PbTiO}_{3} / \mathrm{SrTiO}_{3}$ superlattices. We look at the behavior of ferroelectric domains in these materials across the ferroelectric phase transition and discover a temperature regime where the DWs change their orientation from walls that lie preferentially within the $\{100\}$ crystallographic planes at room temperature to predominantly $\{110\}$ DWs at higher temperatures, with a temperature range that depends on the ferroelectric layer thickness and domain period. Using a nanofocused beam, we obtain maps of the dominant local orientations of the buried ferroelectric domains. Imaging the domain orientations near the edge of the superlattice and in the vicinity of a microscopic defect reveals that DWs tend to align preferentially along the film edges and features associated with the structure of the defect. Furthermore, we obtain local three-dimensional maps of reciprocal space at different points around one of these features and use them to reconstruct images of the local lattice parameter, strain, and strain gradient distributions that are likely to be responsible for the observed DW alignments. Our findings demonstrate that the orientation of $180^{\circ}$ DWs can be controlled by varying the temperature, film thickness, and topographical features of the samples and suggest that patterning features by conventional photolithography or focused ion beam techniques could offer additional new pathways for manipulating DWs for nanodevices [17,18].

$\mathrm{PbTiO}_{3} / \mathrm{SrTiO}_{3}$ superlattices with epitaxial top and bottom $\mathrm{SrRuO}_{3}$ electrodes were grown on $\mathrm{SrTiO}_{3}$ substrates 
using off-axis radio-frequency magnetron sputtering. Here we report the results for two superlattices with compositions $\left(\mathrm{PTO}_{5} \mid \mathrm{STO}_{4}\right)_{28}$ and $\left(\mathrm{PTO}_{8} \mid \mathrm{STO}_{4}\right)_{19}$, where the notation $\left(\mathrm{PTO}_{n_{P}} \mid \mathrm{STO}_{n_{S}}\right)_{N}$ refers to $n_{P}$ unit cells (u.c.) of $\mathrm{PbTiO}_{3}$ and $n_{S}$ u.c. of $\mathrm{SrTiO}_{3}$ per period, repeated $N$ times. Further details of the sample preparation can be found in Supplemental Material [19]. The $\left(\mathrm{PTO}_{8} \mid \mathrm{STO}_{4}\right)_{19}$ superlattice was patterned into $120-\mu \mathrm{m}$-size squares and etched fully down to the bottom electrode using argon ion milling, leaving only the square capacitor structures ( 100 nm thick). Atomic force microscopy (AFM) measurements on the surface of one capacitor revealed a number of micron-sized topographical features that might be regions which were partially etched during argon milling or other extended structural defects in the substrate and/or film. These features, along with the edges of the square capacitor structures, allowed us to study the impact of topography variations and structural defects on DW orientations.

$\mathrm{X}$-ray diffraction experiments were performed at beam line ID01 at ESRF. For temperature-dependent measurements, an incoherent x-ray beam [approximately $50 \mu \mathrm{m} \times 50 \mu \mathrm{m}$ full width at half maximum (FWHM)] was used to probe the average domain behavior. The spatial variation of the domain intensities was studied by focusing the beam with a Fresnel zone plate (achieving a beam size of approximately $60 \mathrm{~nm} \mathrm{FWHM)}$ with the sample mounted on a resistive heater on a piezo stage. The setup at beam line ID01 has been described in detail in Refs. [20-23]. The energy of the incident $\mathrm{x}$-ray beam was tuned to $8 \mathrm{keV}$ using a $\mathrm{Si}(111)$ double crystal monochromator, and diffraction patterns were recorded with a Maxipix photon-counting detector [24]. The spatial dependence of lattice parameters was calculated using the strain and orientation calculation software package X-sOCs [20,25].

Figure 1 shows in-plane reciprocal space maps (RSMs) around the 002 Bragg peak of a $\left(\mathrm{PTO}_{5} \mid \mathrm{STO}_{4}\right)_{28}$ superlattice as a function of the temperature (here and throughout, we label the reflections according to the average perovskite lattice parameter $\sim 4 \AA$; thus, in this case the 002 reflection corresponds to the 0018 superlattice peak). The kink in tetragonality corresponding to the ferroelectric-to-paraelectric phase transition in this superlattice was found to occur at $T_{C} \simeq 570 \mathrm{~K}$. The in-plane RSMs are plotted in reciprocal lattice units (r.l.u.) of the substrate $\left(1\right.$ r.l.u $\left.=2 \pi / 3.905 \AA^{-1}\right)$ and were obtained by integrating the diffracted intensity along the out-of-plane direction in reciprocal space over the range $\Delta L= \pm 0.03$ r.l.u. around the superlattice Bragg peak. The ring of diffuse scattering around the superlattice Bragg peak is due to the ferroelectric domains in $\mathrm{PbTiO}_{3}$ with a periodicity $\Lambda_{d}=3.905 \AA / \Delta H \simeq 5 \mathrm{~nm}$ at room temperature [9].

At $300 \mathrm{~K}$, a diffuse ring of intensity is observed. The intensity distribution, however, is not uniform around the ring, with the highest intensity observed at points where the ring crosses the $H$ and $K$ axes, indicating that DWs preferentially lie in the $\{100\}$ crystallographic planes. As the temperature is increased to $375 \mathrm{~K}$, the ring becomes

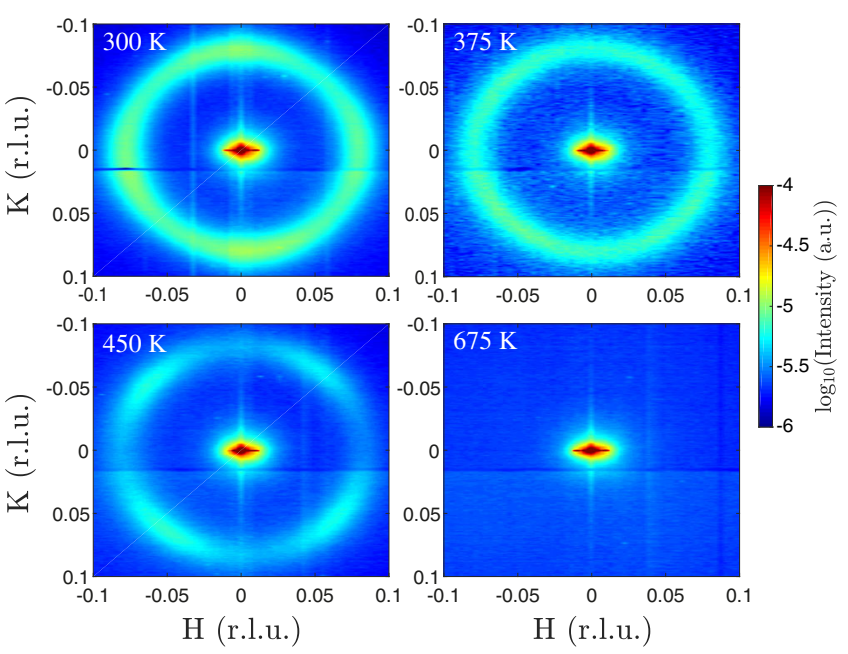

FIG. 1. In-plane RSMs around the 002 superlattice Bragg peak. The ring of intensity is due to diffuse scattering from periodic ferroelectric domains, with a period $\Lambda_{d} \simeq 5 \mathrm{~nm}$. The domain structure evolves from walls lying predominantly in the $\{100\}$ planes at room temperature, to a more isotropic configuration at $375 \mathrm{~K}$ (uniform ring), to $\{110\} \mathrm{DWs}$ at $450 \mathrm{~K}$. No diffuse scattering is observed in the paraelectric phase at $675 \mathrm{~K}$.

more uniform. At still higher temperatures (e.g., $450 \mathrm{~K}$ ), the dominant intensity in the diffuse scattering is redistributed towards four spots with $H= \pm K$, indicating that DWs lie preferentially in the $\{110\}$ planes. The bottom right figure shows the same RSM in the paraelectric phase, where the polarization vanishes and domains disappear. By looking at the intensity as a function of azimuthal angle (Supplemental Fig. S1 [19]), we can clearly see that the $\{100\}$ DWs are dominant at room temperature, whereas the $\{110\}$ DWs are dominant above approximately $375 \mathrm{~K}$.

There are two contributions to the observed intensity changes with increasing temperature: (i) The intensity of the diffuse scattering from the domain structure is reduced due to the reduction of the spontaneous polarization, and (ii) there is a continuous change in the volume fraction of the two orientations as the $\{100\}$ DWs rotate to lie within the $\{110\}$ planes. To separate the first effect, we have measured the $c$ and $a$ lattice parameters at each temperature and normalized the domain satellite intensities by the corresponding $(c / a)-$ $(c / a)_{\text {para }}$ [where $(c / a)_{\text {para }}$ is the tetragonality in the paraelectric phase], which is expected to be proportional to the square of the spontaneous polarization within the domains $[26,27]$ and approximately proportional to the satellite intensity [28]. We then find that the normalized intensity of the $\{110\}$ DWs increases with the temperature, whereas the $\{100\}$ intensity decreases (Fig. 2). This leads us to conclude that there is a gradual reduction in the volume fraction of $\{100\}$ DWs as they reorient towards the $\{110\}$ planes. This conclusion is corroborated by the observation that the in-plane correlation length of the domain satellites along the $\langle 110\rangle$ directions increases with an increasing temperature (whereas it decreases along other directions), signaling an increase in order along $\langle 110\rangle$. 


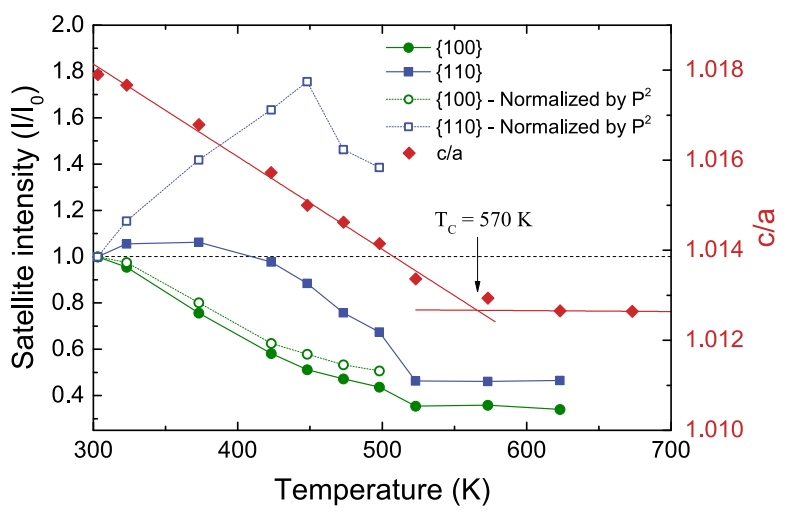

FIG. 2. Average tetragonality $(c / a)$ and domain satellite intensities for the two DW orientations normalized by the values at room temperature. Hollow markers correspond to intensities first normalized by the square of the polarization.

The same reorientation was also observed for samples with different layer periodicities (not shown). The temperature range of the reorientation was found to depend on the domain period and ferroelectric layer thickness, with $\{110\}$ DWs being more pronounced (observed to be dominant across a wider range of temperatures) for samples with thinner layers of $\mathrm{PbTiO}_{3}$ (and hence smaller domain periods).

We note that these observations differ from those of Fong et al. [28], who found that, in ultrathin $\mathrm{PbTiO}_{3}$ films, the diffuse scattering due to the periodic domain structure instead evolves from a uniform ring ( $F_{\beta}$ phase) at lower temperatures to a structure with $\{100\}$ DWs ( $F_{\alpha}$ phase) upon heating, accompanied by an abrupt decrease in the domain period. We also observe a gradual decrease in the domain period with the temperature, but no abrupt changes, consistent with previous reports $[29,30]$. Our observations are also different from the abrupt change in the alignment of ferroelastic DWs observed on heating in $\mathrm{BaTiO}_{3}$ thin films [31].

First-principles simulations of $\mathrm{PbTiO}_{3} / \mathrm{SrTiO}_{3}$ superlattices strained to $\mathrm{SrTiO}_{3}$ have shown that the two domain orientations are very close in energy [32], consistent with the above experimental observation of ringlike features in diffuse scattering from domains in superlattices and the meandering DWs in thicker $\mathrm{PbTiO}_{3}$ films observed by scanning probe techniques in Refs. [33,34]. Model Hamiltonian calculations predict that increasing the compressive strain induces a change from $\{100\}$ to $\{110\}$ DWs concomitant with a structural transition from a phase with nonzero in-plane polarization components to one with purely out-of-plane polarized domains [35]. Although the temperature dependence of the DW orientation was not explicitly studied in Ref. [35], since the same phase transition is accessible by increasing the temperature, a similar DW reorientation might be expected on heating, as indeed observed experimentally here. The prediction that thinner films favor $\{110\}$ DWs [35] is also consistent with our observations.

Since both thickness reduction and temperature increase are expected to broaden DWs [36], we speculate that the DW width plays an important role in the observed DW reorientation. The multicomponent nature of the order parameter in ferroelectrics also means that changes in the DW character (e.g., the effect of in-plane polarization components) [32,37-40] and chirality are also likely to influence both the DW width [41] and preferred orientation; further experimental and theoretical work is needed to clarify this issue.

In order to gain a more local picture of the DW orientations, we use a nanofocused beam to measure the domain satellite intensities for a $\left(\mathrm{PTO}_{8} \mid \mathrm{STO}_{4}\right)_{19}$ superlattice (with domain period $\Lambda_{d} \simeq 7 \mathrm{~nm}$ ) as a function of the position on the sample by translating it relative to the beam using a piezo stage. The resulting maps of the domain satellite intensity for (010) and (100) DWs are shown in Figs. 3(a) and 3(b), respectively. Comparing the two images (see, for example, the features in the square boxes) reveals that some regions are anticorrelated with maxima in the intensity of (100) DWs corresponding to minima in the intensity of (010) and vice versa. Other regions in the images are not fully anticorrelated, presumably because they correspond to regions with similar fractions of both orientations or with DWs that are oriented along other directions.

The maximum intensity variation across the images is around $30 \%$, indicating that both domain orientations are present within the beam footprint at each point, and therefore the patchy features in the images correspond to local variations of the predominant DW orientations rather than individual regions with a specific, well-defined DW
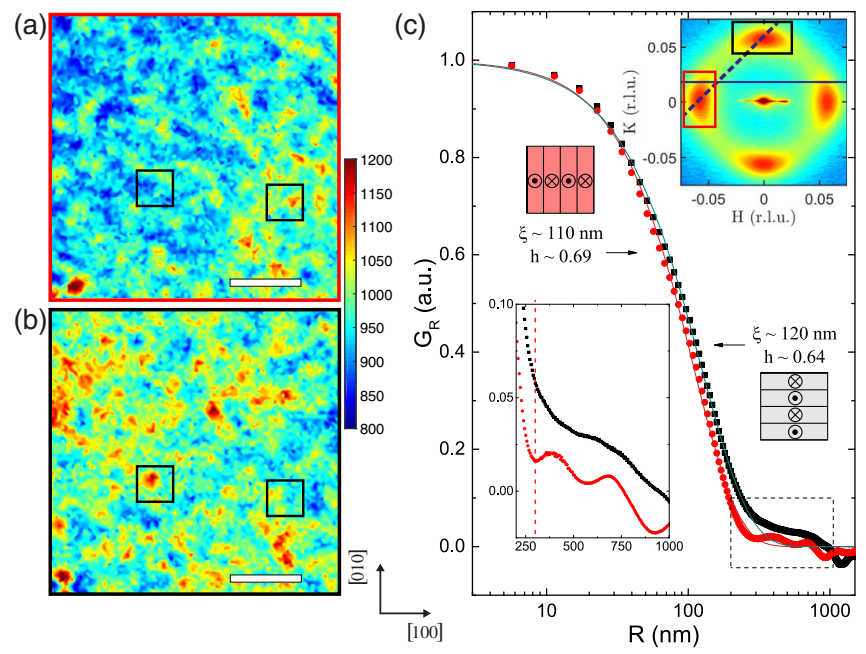

FIG. 3. Spatial variation of domain satellite intensity for (010) (a) and (100) (b) DWs. Horizontal scale bars correspond to $1 \mu \mathrm{m}$. The black boxes show two anticorrelated regions. (c) Normalized average autocorrelation functions for images in (a) (red circles) and (b) (black squares). Fitting parameters $\xi$ and $h$ are discussed in the main text. The bottom left inset is an expanded plot around the dashed box in the main figure, showing a minimum at $300 \mathrm{~nm}$ for one DW orientation. The top right inset shows the in-plane RSM around the 003 peak of the superlattice (unfocused beam). The dashed blue line indicates the approximate orientation of the detector in reciprocal space, whereas the red and blue boxes indicate the domain satellites used for mapping the intensity in (a) and (b), respectively. 
orientation. A lower bound on the size of the latter can be determined from the in-plane correlation length obtained from a Scherrer-type analysis of the FWHM of the domain satellites (measured using an unfocused beam), which we estimate to be around $30 \mathrm{~nm}$. This is several times smaller than the footprint of the focused beam and thus below the resolution of the local measurements.

To estimate the characteristic length scale $\xi$ for the features in Figs. 3(a) and 3(b), we calculate the autocorrelation function (ACF) of each image. If $I(\mathbf{x})$ is the satellite intensity at position $\mathbf{x}$, the $2 \mathrm{D}$ ACF is defined as $G(\mathbf{R})=$ $\langle I(\mathbf{x}) I(\mathbf{x}-\mathbf{R})\rangle$, where $\mathbf{R}$ is a displacement vector and averaging is performed over all possible $\mathbf{x}$. For simplicity, we assume an isotropic distribution of regions with a given DW orientation and use the $2 \mathrm{D}$ ACF to calculate the azimuthal average over all in-plane directions $G(R)$ with $R=|\mathbf{R}|$. To extract $\xi$, we fit $G(R)$ to

$$
G(R)=\sigma_{I}^{2} \exp \left[(-R / \xi)^{2 h}\right] .
$$

This expression [42] has previously been used to estimate domain sizes measured using piezoresponse force microscopy $[43,44]$. In the above, the function $I(\mathbf{x})-I(\mathbf{x}-\mathbf{R})$ is assumed to be a Gaussian random variable with a standard deviation $\sigma_{I}$, and $h$ is a parameter that describes the abruptness of intensity changes ( $h$ is small for very abrupt changes, whereas it tends to unity for smoother variations) [42].

The average ACFs corresponding to the images in Figs. 3(a) and 3(b) (after the subtraction of the mean intensity of each image) are shown in Fig. 3(c). Fitting the autocorrelation data, we estimate the characteristic length scale $\xi$ to be of the order of $110-120 \mathrm{~nm}$, which is comparable to the beam footprint. We note that the length scale extracted from Eq. (1) is always a lower estimate of the feature size [45] and is usually quoted in cases where there is a distribution of sizes. In images with a well-defined feature size, a better measure of this size is the first minimum in the ACF [46]. This minimum is discernible for only one orientation [Fig. 3(a)] and occurs at around $300 \mathrm{~nm}$.

Performing the same analysis on images from other parts of the sample shows that $\xi$ varies significantly from around $100 \mathrm{~nm}$ to microns, and, even within individual images, different length scales are apparent on visual inspection. We therefore conclude that, although the domain period is well defined by the electrostatic boundary conditions, as can be seen from the sharpness of the peaks in the RSM in Fig. 3(c), the dominant DW orientation exhibits variations over a wide range of length scales across the sample and the local DW orientation is likely to be determined by other factors, as we discuss next.

To better understand what influences the local DW orientation, we look at a region of the film close to a topographical defect. The defect has an irregular shape, as seen from the AFM image in Fig. 4(a), and gives rise to long, streaky features of reduced intensity in the map of the superlattice Bragg peak intensity [Fig. 4(a), bottom right]. Intensity maps of the domain satellites reveal that the domain stripes align preferentially along the length of these features. The intensity in these aligned regions is more than double that in the more uniform parts of the film, showing a strong local preference for that particular DW orientation compared to the rest of the superlattice. Local in-plane RSMs, obtained with the nanofocused beam and shown in Supplemental Fig. S2 [19], further highlight this preferential alignment. We also observe some preferential DW alignment with the Ar-ion-milled edges of the square capacitor structures (see Supplemental Fig. S3 [19]).

Furthermore, by obtaining a three-dimensional RSM at each point, we can map the variation of the out-of-plane lattice parameter of the film around this region and extract the local distribution of the average out-of-plane strain $\epsilon_{33}$ that accompanies the DW alignment. The corresponding map of $\epsilon_{33}$ is shown in Fig. 4(b), together with an AFM scan of the local superlattice topography [Fig. 4(c)]. We can further use the strain map to extract the strain gradients $\nabla_{x y} \epsilon_{33}\left(\nabla_{x y}=\mathbf{i}(\partial / \partial x)+\mathbf{j}(\partial / \partial y)\right)$ around this region [Fig. 4(d)]. We find that the regions with preferential

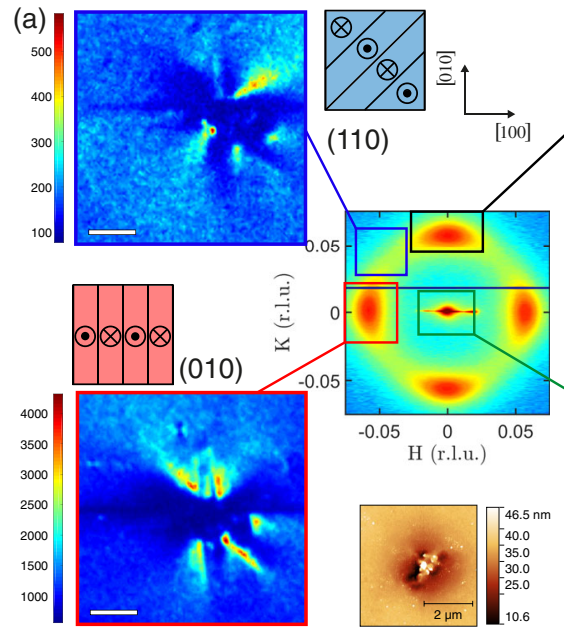

(b) $\epsilon_{33}$

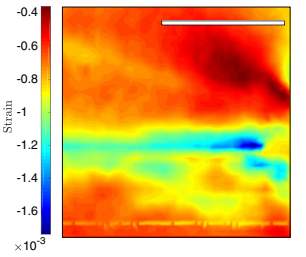

(c)

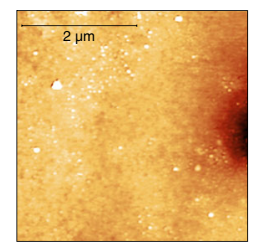

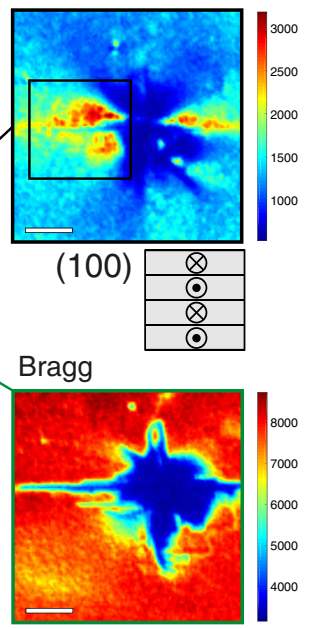

(d) $\nabla_{x, y} \epsilon_{33}$

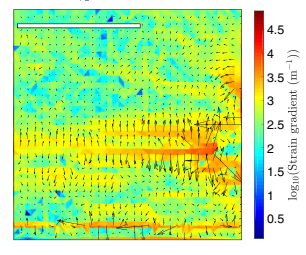

FIG. 4. (a) Domain satellite intensity around a defect in the superlattice, showing DW alignment parallel to the long features of the defect. The schematics show the probed DW orientation for each image, with the central RSM indicating the RS regions probed in each map. The bottom right image is a map of the Bragg peak intensity around the same region. The color bars show the intensity in arbitrary units. The central bottom figure shows an AFM scan of the topography at the center of the defect. (b) Map of the strain $\epsilon_{33}$ in the region marked by the box in the top right image of (a). (c) AFM image of the superlattice topography around the same region. (d) Map of the 2D strain gradient $\nabla_{x, y} \epsilon_{33}$ with arrows and colors representing its direction and magnitude, respectively. The arrows around the region with the largest strain gradient point to a direction perpendicular to the DWs. The horizontal scale bars in all images have a size of $2 \mu \mathrm{m}$. 
domain wall alignment are correlated with changes in strain of the order of $0.1 \%$ and strain gradients of the order of $10^{4} \mathrm{~m}^{-1}$ directed perpendicular to the DWs, as shown by the arrows in Fig. 4(d).

Preliminary studies of the temperature dependence also reveal that the aligned DWs around the defect return to the same configuration when the superlattice is heated above its transition temperature and subsequently cooled to room temperature. The preferential DW alignments reported here are in line with previous observations that DWs in ultrathin $\mathrm{PbTiO}_{3}$ films align with the crystallographic steps of the substrate $[9,28,33,47]$. The precise mechanism for this alignment remains to be fully understood, but our findings suggest that, by nanopatterning the films, conventional lithography methods could be used to define local DW orientation in ferroelectric thin films.

In conclusion, we have studied the behavior of ferroelectric domains in $\mathrm{PbTiO}_{3} / \mathrm{SrTiO}_{3}$ superlattices with synchrotron $\mathrm{x}$-ray diffraction. We discover a temperature regime where the DW normals rotate from preferential $\langle 100\rangle$ alignment at low temperatures to $\langle 110\rangle$ at high temperatures. By probing the spatial distributions of the domain orientations using a nanofocused beam, we were able to map the local orientations of buried domains in these superlattices. We observe that in the vicinity of a structural defect the DWs are preferentially aligned along specific directions and map out the inhomogeneous strain and strain gradient fields associated with this defect. A similar preferential DW alignment is observed along the ion-milled edges of the film, offering a route to controlling the behavior and orientation of domains in ferroelectric thin films that may help further advance the growing field of DW nanoelectronics.

This work was supported by the EPSRC [Grants No. EP/M007073/1 (P. Z.) and No. 1447654 (M. H.)], the A. G. Leventis Foundation (M. H.), and the UCL-ESRF Impact studentship scheme (E. Z.). We acknowledge the European Synchrotron Radiation Facility and the ID01 beam line staff for support during the experiment.

*marios.hadjimichael.14@ucl.ac.uk

[1] G. Catalan, J. Seidel, R. Ramesh, and J. F. Scott, Rev. Mod. Phys. 84, 119 (2012).

[2] E. Salje and H. Zhang, Phase Transitions 82, 452 (2009).

[3] Q. M. Zhang, H. Wang, N. Kim, and L. E. Cross, J. Appl. Phys. 75, 454 (1994).

[4] A. M. Bratkovsky and A. P. Levanyuk, Phys. Rev. B 63, 132103 (2001).

[5] P. Zubko, J. C. Wojdeł, M. Hadjimichael, S. FernandezPena, A. Sené, I. Luk'yanchuk, J.-M. Triscone, and J. Íñiguez, Nature (London) 534, 524 (2016).

[6] J. Seidel et al., Nat. Mater. 8, 229 (2009).

[7] S. Farokhipoor and B. Noheda, Phys. Rev. Lett. 107, 127601 (2011).

[8] S. Farokhipoor et al., Nature (London) 515, 379 (2014).
[9] S. K. Streiffer, J. A. Eastman, D. D. Fong, C. Thompson, A. Munkholm, M. V. Ramana Murty, O. Auciello, G. R. Bai, and G. B. Stephenson, Phys. Rev. Lett. 89, 067601 (2002).

[10] E. D. Specht, H. M. Christen, D. P. Norton, and L. A. Boatner, Phys. Rev. Lett. 80, 4317 (1998).

[11] V. A. Stephanovich, I. A. Luk'yanchuk, and M. G. Karkut, Phys. Rev. Lett. 94, 047601 (2005).

[12] P. Zubko, N. Jecklin, A. Torres-Pardo, P. Aguado-Puente, A. Gloter, C. Lichtensteiger, J. Junquera, O. Stéphan, and J.-M. Triscone, Nano Lett. 12, 2846 (2012).

[13] P. Zubko, N. Stucki, C. Lichtensteiger, and J.-M. Triscone, Phys. Rev. Lett. 104, 187601 (2010).

[14] J. Y. Jo, P. Chen, R. J. Sichel, S. J. Callori, J. Sinsheimer, E. M. Dufresne, M. Dawber, and P. G. Evans, Phys. Rev. Lett. 107, 055501 (2011).

[15] E. Bousquet, M. Dawber, N. Stucki, C. Lichtensteiger, P. Hermet, S. Gariglio, J.-M. Triscone, and P. Ghosez, Nature (London) 452, 732 (2008).

[16] A. K. Yadav et al., Nature (London) 530, 198 (2016).

[17] J. R. Whyte, R. G. P. McQuaid, P. Sharma, C. Canalias, J. F. Scott, A. Gruverman, and J. M. Gregg, Adv. Mater. 26, 293 (2014).

[18] J. R. Whyte and J. M. Gregg, Nat. Commun. 6, 7361 (2015).

[19] See Supplemental Material at http://link.aps.org/ supplemental/10.1103/PhysRevLett.120.037602 for more information on the film growth and characterization, the azimuthal analysis of the domain satellite intensity and reciprocal space maps at different points on the sample with a nanofocused beam.

[20] G. A. Chahine et al., J. Appl. Crystallogr. 47, 762 (2014).

[21] M. Keplinger, B. Mandl, D. Kriegner, V. Holý, L. Samuelsson, G. Bauer, K. Deppert, and J. Stangl, J. Synchrotron Radiat. 22, 59 (2015).

[22] G. A. Chahine, M. H. Zoellner, M.-I. Richard, S. Guha, C. Reich, P. Zaumseil, G. Capellini, T. Schroeder, and T. U. Schülli, Appl. Phys. Lett. 106, 071902 (2015).

[23] S. J. Leake, V. Favre-Nicolin, E. Zatterin, M.-I. Richard, S. Fernandez, G. Chahine, T. Zhou, P. Boesecke, H. Djazouli, and T. U. Schülli, Mater. Des. 119, 470 (2017).

[24] C. Ponchut, J. M. Rigal, J. Clément, E. Papillon, A. Homs, and S. Petitdemange, J. Instrum. 6, C01069 (2011).

[25] D. Naudet, XSOCS software package, 2017.

[26] C. Lichtensteiger, J. M. Triscone, J. Junquera, and P. Ghosez, Phys. Rev. Lett. 94, 047603 (2005).

[27] M. Dawber, N. Stucki, C. Lichtensteiger, S. Gariglio, P. Ghosez, and J.-M. Triscone, Adv. Mater. 19, 4153 (2007).

[28] D. D. Fong, G. B. Stephenson, S. K. Streiffer, J. A. Eastman, O. Auciello, P. H. Fuoss, and C. Thompson, Science 304, 1650 (2004).

[29] P. Zubko, N. Jecklin, N. Stucki, C. Lichtensteiger, G. Rispens, and J.-M. Triscone, Ferroelectrics 433, 127 (2012).

[30] A. Boulle, I. C. Infante, and N. Lemée, J. Appl. Crystallogr. 49, 845 (2016).

[31] A. S. Everhardt, S. Matzen, N. Domingo, G. Catalan, and B. Noheda, Adv. Electron. Mater. 2, 1500214 (2016).

[32] P. Aguado-Puente and J. Junquera, Phys. Rev. B 85, 184105 (2012).

[33] C. Thompson et al., Appl. Phys. Lett. 93, 182901 (2008).

[34] C. Lichtensteiger, S. Fernandez-Pena, C. Weymann, P. Zubko, and J. M. Triscone, Nano Lett. 14, 4205 (2014).

[35] Z. Jiang, R. Zhang, D. Wang, D. Sichuga, C. L. Jia, and L. Bellaiche, Phys. Rev. B 89, 214113 (2014). 
[36] I. A. Luk'yanchuk, L. Lahoche, and A. Sené, Phys. Rev. Lett. 102, 147601 (2009).

[37] J. B. J. Chapman, A. V. Kimmel, and D. M. Duffy, Phys. Chem. Chem. Phys. 19, 4243 (2017).

[38] J. C. Wojdeł and J. Íñiguez, Phys. Rev. Lett. 112, 247603 (2014).

[39] P. Marton, V. Stepkova, and J. Hlinka, Phase Transitions 86, 103 (2013).

[40] D. Lee, R. K. Behera, P. Wu, H. Xu, Y. L. Li, S. B. Sinnott, S. R. Phillpot, L. Q. Chen, and V. Gopalan, Phys. Rev. B 80, 060102 (2009).

[41] B. Houchmandzadeh, J. Lajzerowicz, and E. K. H. Salje, J. Phys. Condens. Matter 3, 5163 (1991).
[42] S. K. Sinha, E. B. Sirota, S. Garoff, and H. B. Stanley, Phys. Rev. B 38, 2297 (1988).

[43] V. V. Shvartsman et al., Appl. Phys. Lett. 86, 202907 (2005).

[44] J. Yao, W. Ge, L. Yan, W. T. Reynolds, J. Li, D. Viehland, D. A. Kiselev, A. L. Kholkin, Q. Zhang, and H. Luo, J. Appl. Phys. 111, 064109 (2012).

[45] C. Mätzler, J. Appl. Phys. 81, 1509 (1997).

[46] S. Fernandez-Peña, C. Lichtensteiger, P. Zubko, C. Weymann, S. Gariglio, and J.-M. Triscone, APL Mater. 4, 086105 (2016).

[47] S. Prosandeev and L. Bellaiche, Appl. Phys. Lett. 91, 072901 (2007). 